\title{
Detection head for radiation measurement in the „water window” region
}

Janusz MIKOŁAJCZYK, Jacek WOJTAS, Rafał RAKOWSKI

Institute of Optoelectronics, Military University of Technology, 2 Kaliskiego St,. 00-908 Warsaw, Poland

\section{Introduction}

Soft $x$-ray radiation is being widely applied in many branches of science and technology. It can be assumed, that this radiation fills the gap between optical- and electron microscopy $[1,2,3]$. Soft $x$-ray technology showed its attractiveness for diagnostics of living cells and biological tissues. The main task of these diagnostics is observation of cells of typical dimensions of $20-40 \mathrm{~nm}$ or even less ones (DNA structures). Currently available diagnostic technologies do not provide a sufficient resolution and request a special preparation procedure of investigated materials. Using the radiation in the range of 2-4 nm (called "water window" region), the described restrictions are minimized.

Analysis of the market shows that there is no commercial available energy meter operated at the water window range. The paper presents an approach of such detection head designed in the Institute of Optoelectronics MUT. The preliminary results of analysis and the experimental investigations of the head are described.

\section{Analyses of the head construction}

For the detection of the energy radiation a detection system is necessary. The sysytem usually consists of optical filters detectors and electronics units It should provide to measure energy radiation with defined uncertainty in the specific wavelength range. The wavelength range is determined by features of optical filters. As the filters, the multilayer mirrors, Fresnel lens or absorption layers could be used. It is worth to notice, that it is very difficult to separate water widow spectrum using single optical element. The efficient filtration can be achieved by using a system of filters. For example the determination of the band-pass filter could be accomplished by two filters operated in the shorter wavelength range and in the longer one.

Analysis of the literature and databases from some research centers show that for the detection system some multilayer mirrors or absorption filters can be appropriate.

The simulation procedure was proposed for designing of the mirrors with high reflectivity in the water window spectrum. The three models of mirrors $(\mathrm{Cr} / \mathrm{Sc}, \mathrm{Cr} / \mathrm{V}, \mathrm{Cr} / \mathrm{Ti})$ were taken into consideration in mentioned above analysis. The results of the simulations are presented in Fig. 1.

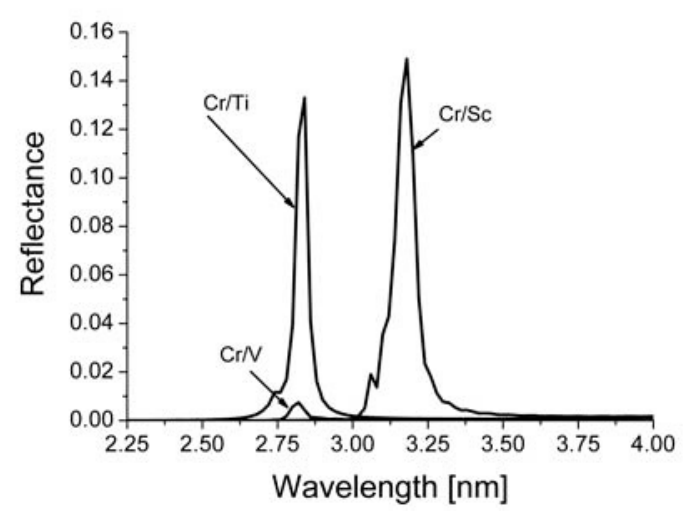

Fig. 1. Reflectance of the simulated multilayer mirrors.

The curves were determined theoretically. Basis on the results the two types of multilayer mirrors were selected for further considerations. The calculations were made together with scientists from Fraunhoffer Institute (Jena, Germany). The conclusions from the results were implemented practically during technological process. The results showed that better parameters of fabricated mirrors achieved for $\mathrm{Cr} / \mathrm{Ti}$ structures. For optimal features of the structure (layer period of $4.235 \mathrm{~nm}$, layers number of 150 , incidence angle of $70^{\circ}$ ), the reflectivity characteristics were determined. The analyzed mirrors show both high reflectivity and spectral selectivity. It is worth to note, that the energy measurements for the whole water window region are not provided because of shortcomings of mirror selectivity. During the next 
analyses the selectivity was diminished at the cost of reflectance decreasing. This goal was obtained by reduction of the mirror layers number.

IFigure 2 shows reflectance characteristics of modified mirrors are presented. It can be noticed, that the maximum of mirrors characteristics changes significantly, while the bandwiths are rather unaltered. The maximum is increased with the number of mirror layers. That is why the multilayer mirrors can be used more effectively for radiation emitted by well-defined source.

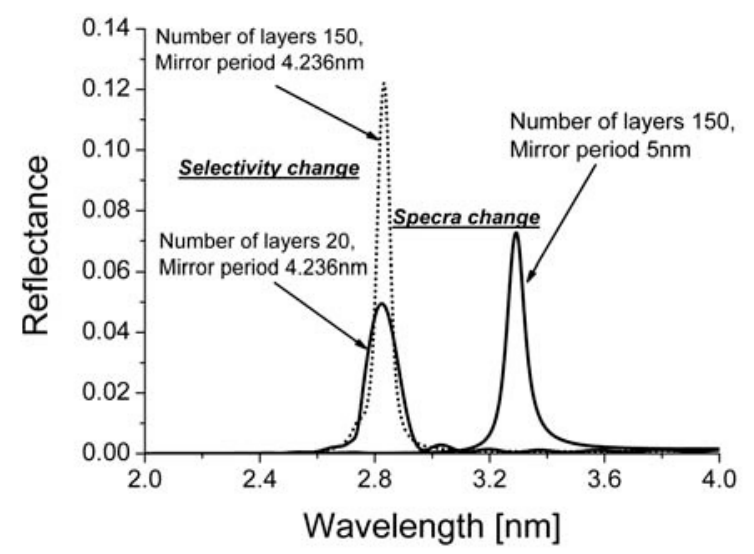

Fig. 2. Reflectance characteristics of $\mathrm{Cr} / \mathrm{Ti}$ modified mirrors.

There is possible to adjust the maximum of reflectivity for specific wavelength range. The mentioned properties of mirrors can be applied in detection system in order to compare the emission lines generated by different radiation sources. At least two mirrors are requested in the analyzed procedure. Nevertheless, the application of the mirrors increases the cost and complicates construction of eventual detection system.

The absorption filters could be an alternative solution for the presented work. The analyses of the filters applicability were made in collaboration with scientists from International Radiation Detectors Inc. and Lebow Company, as well. In practice, the realization of the absorbing filter with very narrow profile of transmission coefficient in specific wavelength range is impossible. Therefore it was decided to design two detection channels working in different wavelength ranges. The radiation with wavelength range less than $1.5 \mathrm{~nm}$ is measured in the first channel. The second channel determines the intensity above the wavelength of $4 \mathrm{~nm}$. The intensity of radiation in the range of water window on basis of measurements of signals in both tracks can be determined by comparison.

In order to find the optimal filter materials, the computer simulations of transmission coefficient were conducted. Results of these simulation showed, that optimum combination will be combining of beryllium filter with thickness of $20 \mu \mathrm{m}$ and aluminum filter with thickness of $0.2 \mu \mathrm{m}$. The profiles of transmission coefficient of two analyzed filters are presented in Fig. 3. Basing on conducted analysis it was decided to apply a set of $\mathrm{Al} / \mathrm{Be}$ filters.

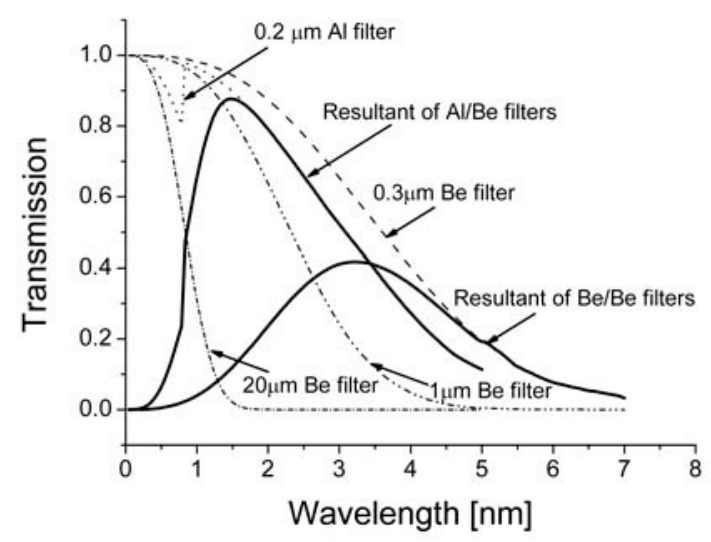

Fig.3. Transmission of some absorption filters. 
Using the described set of the filters, the measurement of radiation energy in the whole considered wavelength range was carried out. The absorption filters also simplify the mechanical construction of instrument design as well as significantly decrease the costs of realization.

Operational features of detection instrument, e.g. sensitivity, range of correct work as well as time-frequency parameters are also determined by detector parameters. For the detection of radiation in the water window spectrum frequently silicon photodiodes are applied. The photodiodes are characterized by quantum efficiency of $100 \%$ in analyzed spectral range. IRD Inc. company specializes in production of this photodiodes. Some spectral characteristics of photodiodes sensitivity also are well fitted to set wavelength ranges. Basing on the IRD Inc. photodiodes data-sheet, the AXUV 100 series were applied in detection head. The conducted investigations of the spectral sensitivity of the available detectors have given the value at the level of Rdet $=0.24 \mathrm{~A} / \mathrm{W}$.

Output signal from detector is proccesed by preamplifier. Voltage, transimpedance or charge integrating preamplifiers are mostly used with photodiodes. With regard to charge evaluating procedure, the integrating preamp was used. The charge sensitivity and equivalent noise charge (ENC) are the most important parametrs of the preamp. The selection of photoreceiver first stage is extremely important. Such selection was made to obtain the maximum of the signal to noise ratio as well as proper frequency range. Developed preamp of detection head was designed basing on two-channel amplifier with gated capacity, ACF 2101 type, Burr-Brown company.

The described analyses make it possible to design a construction of the detection head. The main head elements is a detector-filter holder. The view of the detection head and the holder is presented in Fig.4.
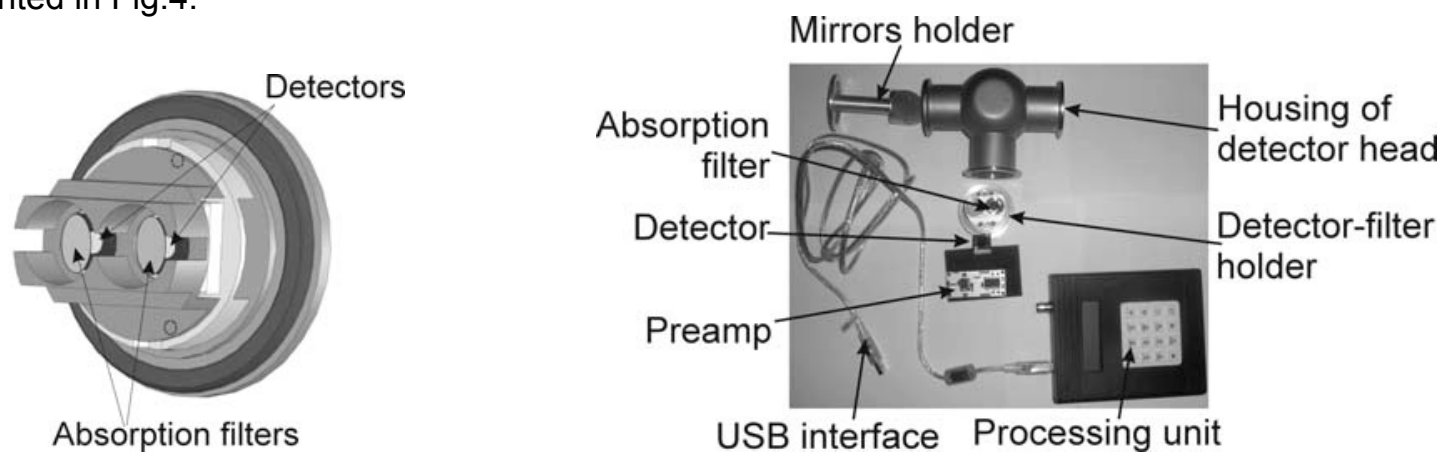

Fig. 4. View of the detection head and detector-filter holder.

\section{Computer simulations of preamp}

Computer simulations of the preamp were done using ICAP4 software. A special attension was paid to investigation of the influence of detector's parameters on detection performance. Charging time of integrating capacitor depends on amplifier features, e.g. input current, input capacity and slew rate. Therefore absolute value of the capacitor should be at considerable value in relation to chosen input current. For the analyzed preamp the frequency band was $500 \mathrm{kHz}$. The application of additional external capacitor results in the band narrowing (see Figure 5).

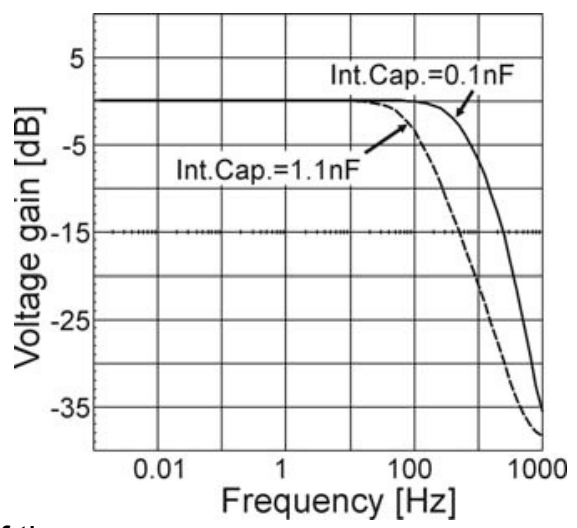

Fig.5. Frequency characteristics of the preamp.

\section{Experimental investigations}

The main aim of the experimental investigations of the detection head was to define the charge sensitivity, frequency band and noise spectrum. The noise spectrum defines threshold value of radiation 
energy, which can be measured by the detection head. For sensitivity investigations of detection head current-voltage source Keithley 236 and generator Agilent 33220A were utilized. Noise spectrum was determined using the phase-sensitive amplifier SR 850 (Lock-In).

During time measurements the influence of integrating capacitor changes on output voltage was determined. The obtained voltage signals are presented in Fig. 6.

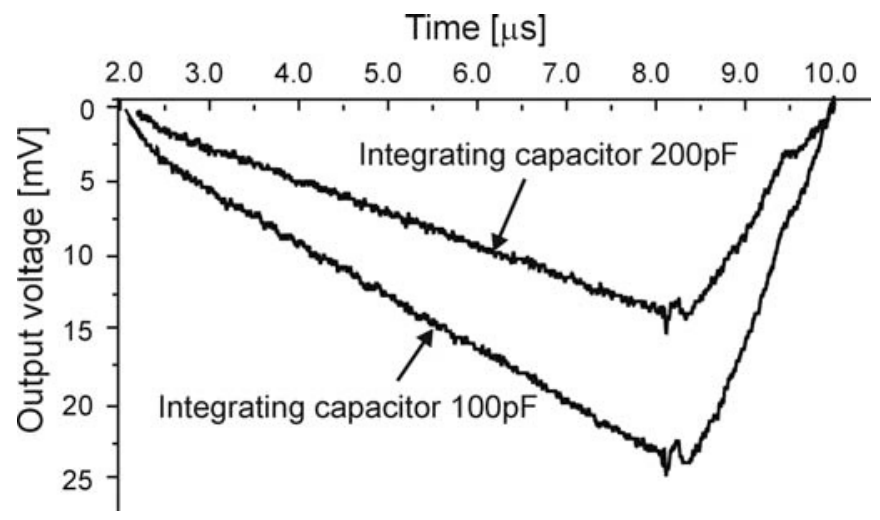

Fig.6. Output signals from preamp for two values of integrating capacitors.

It was noticed, that it is evidently increase of value of integrating capacity entails simultaneous increasing in fall time of (RESET ON) signal. The measured values of fall time are characterized by linear dependence of the capacity. The maximum operation frequency of $97 \mathrm{kHz}$ was received for $100 \mathrm{pF}$ internal capacity of ACF 2102. Exceeding this value, output signals start overlapping and constant component of the signals is extended.

The presented signals make it possible to define preamp charge sensitivity. It is shown the sensitivy of the preamp strongly depends on value of integrating capacity. Knowing the current value produced by the source, capacity of charge preamp and charging time, the charge sensitivity of the preamp was calculated. For value of $100 \mathrm{pF}$, the charge sensivity was $10.8 \cdot 10^{6} \mathrm{~V} / \mathrm{C}$. The sensitivity is inversely proportional to the integrating capacity.

Measurement of noise parameters included obtaining some spectral characteristics of output noise voltage. In received spectra, the two regions can be distinguished. In these regions predominate noises: thermal and of $1 / f$ type (see Figure 7 ).
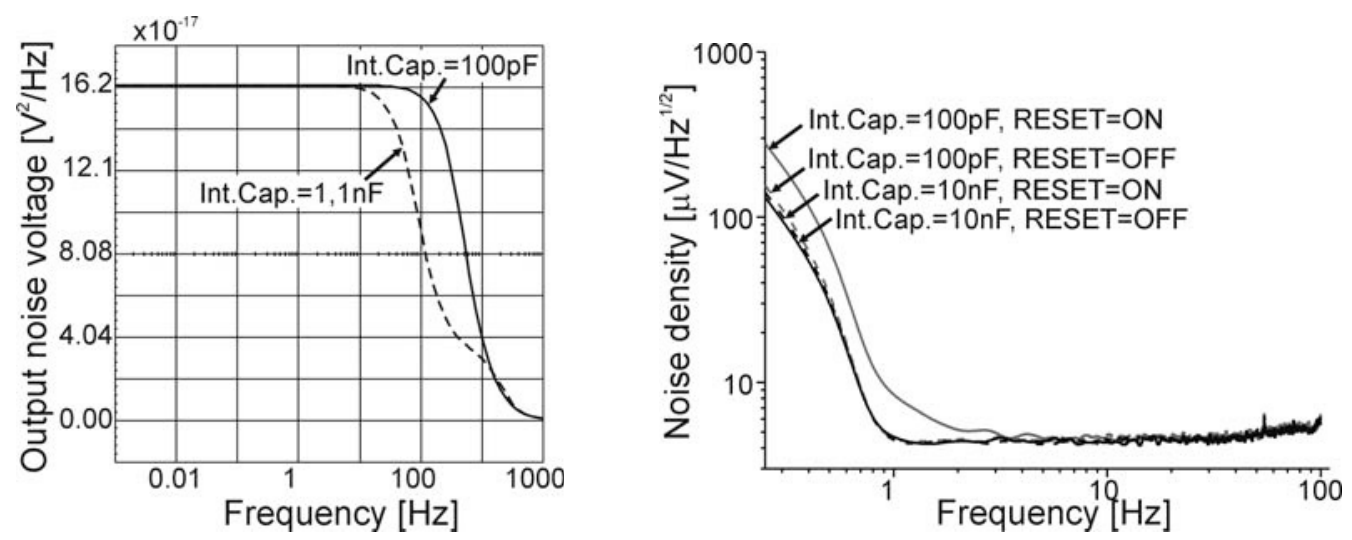

Fig. 7. Noise features of the preamp [8].

Increasing operation frequency (above value of $1 \mathrm{kHz}$ ) it is possible to limit the noise to the value of $4.5 \mu \mathrm{V}$. Simultaneously growth of integrating capacity value causes the decrease of noise voltage (especially for $1 / \mathrm{f}$ noise type). Change of amplifier operation mode (integrate-reset) influences on noise spectra. For integration operation mode the noise voltage is higher than in reset process. Assuming charge sensitivity value of $10.8 \cdot 10^{6} \mathrm{~V} / \mathrm{C}$ and noise voltage of $4.5 \mu \mathrm{V}$, equivavlent noise charge was of $0.049 \mathrm{nC}$. For silicon detector, the noise charge is equivalent to the measured radiation energy in the water window wavelength range of $0.2 \mathrm{~nJ}$.

\section{Summary}

Described detection head is dedicated to radiation energy measurements in the range of water window radiation. This instrument can be used in preliminary investigation of radiation source efficiency to be 
applied in the microscopy. Basing on determined head parameters the sensitivity was estimated according to formula: $R_{G}=R_{d e t} k_{q} T_{\text {filtr }}$.

The calculated sensitivity was $1.3 \mathrm{MV} / \mathrm{J}$. Essential advantages of described detection head are: simple construction, low price and automated measuring procedure. User is able to change head sensitivity by exchange of integrating capacitor. Further works will concern realization of series of calibration of the developed head.

\section{ACKNOWLEDGEMENT}

The researches are financed by Research Grants from the Polish Ministry of Science and Higher Education, No O N515 005043.

\section{LITERATURE}

[1] Adam J.F., Moy J.P., Susini J., Table-top water window transmission x-ray microscopy: Review of the key issues, and conceptual design of an instrument for biology, review of scientific instruments 76, (2005), 091301

[2] Artyukov LA., Vinogradov A.V., Kas'yanov Yu.S., Savel'ev S.V., X-ray microscopy in the carbon window region, Quantum Electronics 34(8) (2004), pp.691-692

[3] TakmanT., Compact Soft X-Ray Microscopy: Sources, Optics and Instrumentation, PhD Thesis, Royal Institute of Technology, Stockholm, Sweden 2007

[4] Takenaka H., Ito H., Nagai K., Muramatsu Y., Gullikson E., Perera R.C.C., Soft X-ray reflectivity and structure evaluation of $\mathrm{Ni} / \mathrm{C} / \mathrm{Ti} / \mathrm{C}$, multilayer $\mathrm{X}$-ray mirrors for water-window region, Nuclear Instruments and Methods in Physics Research A,467-468 (2001) pp.341-344

[5] http://henke.Ibl.gov/optical_constants/

[6] Yulin S., dane z symulacji, Fraunhofer Institut Angewandte Optik und Feinmechanik

[7] Mikołajczyk J., Bielecki Z., Application of laser-plasma source with gas-puff target for calibration of EUV radiation detectors, Elektronika, nr 12, (2007), pp.51-53

[8] Mikołajczyk J., Bielecki Z., Nowakowski M., Wojtasj., Głowica detekcyjna z układem całkowania synchronicznego. Elektronika, 2008, nr 11, pp. 133-136.-in polish. 